\title{
DE LA MATEMÁTICA, DE LA EDUCACIÓN Y SU ENSEÑANZA
}

\author{
Javier de LORENZO
}

Al hablar de Matemáticas y de su Enseñanza parece que nos ponemos unas anteojeras: sólo vemos el rótulo Matemática. Algo común en otros campos y que lleva a escindir los saberes en, por ejemplo, Científicos y Humanísticos atribuyendo a cada uno papeles muy diferenciados según valoraciones muy arraigadas pero no por ello menos improcedentes. Desde estas valoraciones se quiere que los saberes científicos no son humanísticos $y$, consecuentemente, se muestran embrutecedores para el común de los hombres, convertidos en hombre-masa, sin rasgos humanísticos. El "científico", mero utilizador de unos métodos, los "científicos", con los que obtener unos resultados, sin que tenga que emplear la imaginación o unas mínimas capacidades creadoras que parecen reservadas exclusivamente para lo humanístico...

Sin embargo, la Matemática es un Hacer y, como tal, una producción y un producto de la especie humana. Como hacer construido por la razón conceptual imaginativa se encuentra incardinado en dicha especie y, más aún, en unos ciertos tipos de sociedad que son los que han posibilitado y posibilitan el mismo. El Hacer matemático no puede desgajarse de la sociedad en la que ese hacer se tiene, por mucho que el producto obtenido en él provoque la ilusión de un saber verdadero, universal y objetivo, modelo por excelencia de la objetividad de los restantes saberes que lo utilizan y que, por ello, simplemente hay que descubrirlo y enseñarlo a los alumnos como un ideal absoluto.

El Hacer matemático está incardinado en una sociedad a la que refleja. Pero también se ha constituido en uno de los modos de producción más potentes 
que ha creado el hombre occidental, modo de producción que posibilita el conocimiento y consecuente transformación y actuación sobre la naturaleza.

Como modo de producción, motor y clave para el desarrollo de una sociedad como la que denominamos occidental, esta parece exigir su manejo por parte de algunos miembros de esa sociedad. Y, de aquí, que el producto matemático, o al menos parte del mismo, deba impartirse en unos ciertos grados de instrucción, a ciertos niveles, a los miembros de la sociedad. Bien entendido que esa enseñanza ha surgido como tal cuando esa sociedad tiene necesidad de los saberes matemáticos en unos determinados niveles.

Es el tipo de estructuración y desarrollo social el que impone, en el fondo, las líneas básicas de lo que hay que enseñar y los legisladores suelen ir a remolque de las "necesidades" que se van imponiendo en la instrucción matemática. Ir a remolque también de quienes se dedican al oficio enseñante, inmersos en unas valoraciones que no son estáticas. Algo que, ciertamente, no sólo ocurre en la disciplina matemática.

Y esto último obliga a una serie de interrogantes: ¿cuándo se impone la necesidad de una enseñanza de la Matemática, de otras materias, y a qué niveles? ¿Y qué función se asigna al aprendizaje de esta disciplina, cuál es su papel o qué se pide de la Matemática en la instrucción?

En otras palabras, ¿Por qué enseñar esta disciplina? Y, aceptado que se deba enseñar, ¿a quiénes y cómo enseñarla?

* La Revolución Americana y, fundamentalmente, la Francesa, constituyen el momento de inflexión para que la sociedad occidental sufra un cambio no sólo cuantitativo sino cualitativo en sus formas, en sus estructuras. Un cambio que conduce a las Revoluciones Industriales y al nacimiento de la Ciencia como tal. Hasta ese momento el Ámbito de estas sociedades era un Ámbito eminentemente Simbólico. En él las Universidades continuaban la tradición enseñante escolástica aunque, frente a ellas, comenzaban a estructurarse algunas Instituciones como las Academias y grupos de "filósofos naturales».

Desde la Revolución Francesa, el estado burgués naciente requiere de nuevos tipos de ciudadanos diferentes al noble, al cura, al viejo soldado. Requiere de ingenieros de caminos, canales y puertos, requiere de ingenieros navales y de armamento, requiere de abogados, médicos, arquitectos... Algo que la nobleza, por sí, no proporciona. Y ello obliga a crear las politécnicas o Escuelas de Ingenieros militares, las Universidades burguesas al nuevo estilo. Obliga a 
que el Estado se preocupe de una Educación o Instrucción que dote a los miembros de la nueva sociedad de los instrumentos necesarios para su desarrollo. Lo cual conlleva la creación, obligada, de los cuerpos de enseñantes: alguien debe enseñar a quienes se condiciona a aprender...

Un tipo de Instrucción, la Superior, aceptada por todos los Estados occidentales en el XIX. Naturalmente, con sus condicionantes: son los gobiernos quienes tratan de controlar el funcionamiento de esas nuevas instituciones, con el nombramiento de profesores, asignación presupuestaria, colocación final... No voy a entrar en detalles de estos controles, de las pugnas que, consecuentemente, se originan - como en España con las distintas Leyes de Instrucción Pública, de Gil de Zárate y posterior Ley Moyano -1857-, los Decretos de Orovio y las expulsiones o nombramientos de Catedráticos según unos u otros Gobiernos... o, en Alemania, las pugnas de Fitche, Hegel con los diversos gobiernos de los landers alemanes, las dificultades para nombrar a un matemático como Dedekind catedrático...-.

Esa Instrucción Superior, escindida en Universidades, Escuelas de Ingenieros, Politécnicas o Academias Militares, exige un propedéutico, una preparación previa para aquellos que van a cursarla. Y ese propedéutico viene dado por los Liceos, Gimnasios, Institutos Técnicos. En ellos se impartirá lo que, en grandes términos, se pueden denominar Enseñanzas Medias. Al ser paso obligado para el ingreso en las Grandes Escuelas, en la Universidad, el contenido o materias que en esta Enseńanza Media se imparta vendrá determinado por el contenido que se imparte en la Universidad, en las Grandes Escuelas. Todo ello avalado por los exámenes de ingreso correspondientes...

El resto de la población, en cuanto a la instrucción o enseñanza, queda al margen. $\mathrm{O}$ es analfabeto o recibe una instrucción elemental, generalmente en manos de órdenes religiosas.

Es de la Universidad, de las Grandes escuelas, de la que salen los cuadros útiles, los nuevos profesionales, para una sociedad como la burguesa del $s$. XIX. Grandes cuadros que suponen, a la vez, una organización estamental muy clara, con la posibilidad de ascender en el estatuto de reconocimiento social en dicho tipo de sociedad.

El s. XIX supone, en el mundo occidental, la Revolución Industrial y ello entraña, evidentemente, la necesidad no sólo de unas colonias, sino de una mano de obra que ha de ir teniendo una mínima cualificación. Por un ejemplo: 
El ferrocarril supone no sólo la fabricación de rieles, máquinas de vapor, vagones...: supone la existencia de unos empleados que expendan unos billetes, que controlen unas mercancías, telegrafistas que sepan comunicarse con otras estaciones... Supone la existencia de unos empleados que, sin ser ingenieros o universitarios, sepan leer, escribir, hacer cuentas...

Y ello obliga a que los distintos estados planteen la necesidad de una educación, de una instrucción mínima general para el buen funcionamiento y desarrollo del mismo. Bien entendido que es una necesidad pragmática que se oculta bajo las ideologías de una mejora del individuo, de su formación, etc. Pero que no hace otra cosa que preparar los instrumentos necesarios para el funcionamiento de un tipo de sociedad.

Problema educativo que se debatirá, ampliamente, a lo largo del siglo con dos posiciones muy claras: la de quienes ven en la bondad del individuo su propia esencia y, por ello, no requiere de instrucción extraña alguna, considerando que esa instrucción lo único que hace es moldear al individuo con las ideas del tipo de sociedad al que va a pertenecer, por lo que se oponen a la implantación de cualquier tipo de enseñanza obligatoria; y la de quienes ven que esa bondad intrínseca no existe y han de ser personas útiles a la sociedad y cuanto más instruidos, más útiles por lo que la enserianza obligatoria se hace imprescindible y necesaria.

En cualquier caso, se van dictando leyes de Instrucción pública en los diversos paises occidentales que adoptan la educación del individuo como clave para la mejora, desarrollo y progreso de la sociedad - siempre, por supuesto, bajo el lema de un «ideal de la humanidad»-. Leyes que, en general, no se cumplen y se deja a los pueblos el pago a los maestros lo que entrana, evidentemente, la nulidad en la aplicación de la ley. Pero es tema del siglo como reflejan las novelas de Dickens, por ejemplo. Y los planes de reformas educativas, los Congresos a que van dando paso...

Tras la guerra franco-prusiana y la implantación de la Tercera República, se dictan en Francia tres leyes que estimo fundamentales: Enseñanza Obligatoria, Enseñanza laica y Creación de las Escuelas Normales. Esta última como clave porque es la creación de las Escuelas de maestros, de los magisterios. Y sin maestros "oficiales" es imposible imponer una enseñanza primaria obligatoria.

Junto a esa instrucción en Artes y Oficios, junto a la Enseñanza Primaria que se quiere obligatoria y común para todos los ciudadanos ya en los últimos 
años del s. XIX, los Liceos, Gimnasios e Institutos Técnicos siguen como los centros clave para el acceso a la Universidad, a las Grandes Escuelas.

La obligatoriedad de una instrucción primaria para todos los ciudadanos y la función que se le asigna viene expresada con radical nitidez en 1910 por un matemático como Félix Klein, autor de un maravilloso libro Matemática elemental desde un punto de vista superior, dedicado precisamente a los profesores de esa instrucción obligatoria elemental: el Estado necesita servidores y no sólo de alto nivel -abogados, politécnicos, médicos, ingenieros... - sino comerciantes, carteros, empleados, oficinistas... que no tienen por qué ser licenciados, ingenieros, politécnicos... pero sí tener una preparación cultural básica para la mayor eficiencia y servicio al estado al que pertenecen.

Se puede esbozar un esquema en el que una persona de una sociedad como la occidental se ha movido, en el último siglo, y en cuanto a la instrucción, de la manera siguiente: una instrucción elemental que llega hasta los catorce anios en el mejor de los casos y a los diez años para quienes se orientan al ingreso en Bachillerato. Estos últimos, superado ese ingreso, cursan Enseñanzas Medias durante unos siete años $y$, tras nuevo examen de ingreso, pasa a la Universidad o a las Escuelas de Ingenieros que le posibilitan no sólo un título académico sino, básicamente profesional.

Por otro lado la Investigación va ligada a las Universidades y a los centros que se ligan con ella: los Institutos Técnicos. Así, a solicitud de Siemens y en función de obtener unos procesos para la obtención de acero más puro, su amigo Hemholtz consigue la creación del Instituto Técnico de Berlín. Orientado a la investigación, en él, Hertz conseguirá la plasmación de la teoría electromagnética con las ondas hoy calificadas de hertzianas. A su vez, en Inglaterra, se crea el Instituto Cavendish dirigido por Maxwell y lord Kelvin, donde Thompson conseguirá detectar el electrón. En Estados Unidos, y en plena guerra civil -1861—, se crea el MIT... Centros de investigación que, aun independientes, se ligan a la Universidad.

Una Universidad a la que se asigna un papel básico de formar no sólo profesionales y profesores para su autoabastecimiento, sino de crear Ciencia. Con una cierta pugna con las Escuelas Superiores de Ingenieros que se ven abocados, de manera casi exclusiva, a una función pragmática: la de ser aplicadores de lo que en esa investigación se obtiene. Lo cual no es del todo punto correcto porque también en esas escuelas se hace investigación. 
En algunos paises como España es en las Escuelas de ingenieros y en las Academias Militares, realmente, donde se está más al tanto de algunas disciplinas científicas que en la propia Universidad, más abocada a cuestiones politicas que de investigación. Bastaría ver cómo en Matemáticas una figura como Echegaray, ingeniero entre otras profesiones, puede estimarse como uno de los grandes introductores de la Matemática del momento.

En este cuadro la Matemática va a tener un papel básico: es el lenguaje o instrumento esencial por el que se desarrolla la ingeniería, la arquitectura, las ciencias en general... Bien entendido que un cierto tipo de Matemática: la apoyada básicamente en el Cálculo infinitesimal junto a un tipo de Geometría como la Descriptiva que terminará calificándose como Dibujo Técnico. Y es en función de estas aplicaciones como ese mismo Hacer matemático se va constituyendo a lo largo del s. XIX. Los Ilustrados habían preparado el terreno y manejado algunas ecuaciones diferenciales, los desarrollos en serie, el cálculo variacional... Y en Francia ha surgido lo que denominar Programa Fourier que potenciará la llamada "matemática aplicada", frente al que se alzará, tímidamente, la "matemática pura", la realizada "por el honor del espíritu humano" y que mostrará, también, su potencia como matemática aplicable a la physis.

Es un Hacer que parte de la magnitud continua enfocada intensionalmente y que no se plantea cuestiones de fundamentación porque su objetivo se centra en resolver problemas aportados por la Filosofía natural, por la Física en su sentido más amplio. Problemas dados ahí, en concreto. Los trabajos de Laplace, Lagrange, los de un Euler, Gauss o Cauchy... van en esta línea. Y es cuando Cauchy ha de crear, realmente, el Análisis matemático junto al Análisis algebraico. Y el Análisis de variable compleja... Un tipo de Hacer que he calificado en otros lugares de Hacer Figural. Se va construyendo parte de la Matemática que, en el fondo, hoy conocemos.

Es una Matemática requerida por el ingeniero, por el arquitecto, por el científico en general y no sólo por el matemático profesional puro. Los condicionantes iniciales -el establecimientos de las Escuelas Politécnicas, Escuelas Superiores...- hacen que a lo largo del siglo XIX se vayan elaborando textos con este tipo de materia y con este enfoque, incluso tablas o formularios con los valores correspondientes a las integrales de algunas funciones... Y es la queja de algunos matemáticos, ya profesionales "universitarios", por el enfoque pragmático de esa Matemática frente a una Matemática "pura»... 
Naturalmente esto plantea algunos problemas en cuanto a lo que se pretenda ensefiar, en cuanto a los contenidos propios para el propedéutico. Si es para el Liceo, Gimnasio o Instituto, la cuestión, muy clara: hay que preparar para el ingreso en la Universidad, en las Escuelas. Y la clave, rudimentos de Cálculo, de resolución de ecuaciones, de trigonometría y algo de geometría métrica euclídea. Pero si es para la Enseñanza Obligatoria, realmente, todo eso sobra. Si se quieren ciudadanos útiles como comerciantes, funcionarios, etc.., lo que importa es que sepan leer, escribir y, en lo referente a la Matemática, unos rudimentos de Geometría y de Aritmética, que sepan calcular lo más correctamente posible ayudándose, para ello, si se requiere, de la máquina de calcular.

Es una escisión en estamentos que todavía en 1933 Puig Adam, con Rey Pastor, expresó nítidamente en las palabras:

Mientras que un maestro de escuela debe considerarse completamente fracasado si sus alumnos salen a la vida sin los pertrechos indispensables que significan saber leer, escribir y calcular correctamente, en cambio un bachillerato que no haya dejado en la memoria de los alumnos indeleblemente grabada para siempre ninguna declinación latina, ninguna fórmula de trigonometría, ninguna especie botánica...

Un tipo de sociedad que condiciona el papel que la Matemática tiene en la misma en función del papel que se le asigna a los distintos tipos de ciudadanos, a los estamentos en los cuáles van a situarse. $\mathrm{Y}$, por supuesto, lo que de esa Matemática debe enseñarse en cada uno de los niveles educativos que esa sociedad establece.

En este siglo, y a partir de los 50, se ha producido un cambio radical en la sociedad occidental, en los modos de producción que la sustentan y condicionan. Se ha pasado a un Ámbito Tecnológico donde la producción y el consumo imponen su valoración máxima. Es un Ámbito en el cual las Grandes Escuelas y las Universidades ya no pueden ser Centros de Investigación en determinadas áreas. No ya una Universidad sino ni siquiera un Estado como el español, el alemán o el francés pueden construir, por su cuenta y riesgo, un acelerador de partículas. El físico que se dedique, por ejemplo, a la Física del estado sólido, no tiene su lugar en la Universidad porque esta no tiene medios suficientes para él. La Medicina depende de otros centros de investigación: empresas, fábricas de productos químicos... La revolución de ordenadores no la ha hecho la Universidad sino la IBM y otras empresas similares junto a la 
presión militar. La Universidad carece de medios para una investigación de punta en muchos terrenos.

A ello se ha sumado otra concepción: la exigencia de una instrucción básica y obligatoria, pero común a todos los individuos hasta los 16 ańos y, en algunos paises, la concepción de una Universidad abierta a todos los ciudadanos. Lo cual supone, por un lado, la no escisión entre propedéutico y enseñanza primaria, sino instrucción básica común pero con una salvedad: con materias optativas, diversificadas desde el origen. Por otro, que ya no son las materias universitarias las que condicionan los programas, los contenidos de la Enseñanza Media, sino las necesidades sociales en general, desde las cuáles se exige su impartición en dicha Enseñanza. Las oficinas y empresas requieren personal "cualificado" pero no respecializado". Enseñanza obligatoria para todos y una serie de disciplinas más o menos difusas, sin contenidos memorísticos, pero que permitan una ulterior colocación y ser útiles a la Sociedad. Posteriormente, unas carreras también muy escindidas y con enfoques más o menos altamente profesionalizadas pero que obligan a una ulterior preparación interna dada ahora por las propias empresas...

* He intentado dar respuesta a una de las primeras preguntas que hice al comienzo: ¡cuándo se impone la enseñanza de la Matemática y a qué niveles? $\mathrm{Y}$, como respuesta, consignar que esa enseñanza se impone a lo largo del s. XIX y como consecuencia de un tipo de sociedad burguesa, de una Revolución Industrial, y en los paises occidentales, en aquellos en los cuáles se produce dicha Revolución Industrial.

También, y haciendo camino, he tratado de responder a una segunda cuestión, aunque ahora de modo parcial: ¿qué función o papel se asigna al aprendizaje de esta disciplina? Y he creído ver que se le asigna, como función básica, la de preparar, en un primer momento, ingenieros, politécnicos, arquitectos, profesores... y también una función de investigación enlazada con las restantes disciplinas de las que, en un primer instante, no aparece desgajada.

En un segundo momento, esa ensefianza se pretende generalizar para que se puedan tener buenos y eficientes servidores del Estado como pueden ser los comerciantes, funcionarios... que deben poder manejar, incluso, las calculadoras para hacer más eficaz su trabajo.

En un tercer momento, y manteniendo los dos estados anteriores, preparar para obtener unos grados medios de nivel inferior al ingeniero, arquitecto... 
pero superior a ese empleado público o de empresa medio: aparición de peritos, aparejadores...

Simultáneo al segundo momento, la convicción de que todo ciudadano debe saber leer, escribir y hacer buenos cálculos aritméticos; y ello porque sobre ese ciudadano va a recaer la función de dar su voto "democrático" a los sucesivos gobiernos que se alteren en los diferentes estados.

$Y$, en momentos como los actuales, una enseñanza que se enfoca como servicio del estado hacia el ciudadano y que obliga a que ese ciudadano, para ser tal y obligatoriamente, pase hasta los 16 años en los centros educativos. Después, continuará o bien en centros educativos superiores o en centros de formación. Sólo algunas Grandes Escuelas y Facultades imponen un cierto «numerus clausus"...

Ello supone, en el fondo, distintos contenidos de matemática: en una enseñanza general obligatoria, la dada antes por los maestros, basta un buen dominio del cálculo y algo de geometría; en un grado intermedio, propedéutica necesaria para los escalones posteriores, el contenido vendrá establecido, precisamente, por dichos escalones; en el superior, se produce una escisión: la de quienes usan o utilizan meramente la Matemática, la de los pocos que se dedican a su cultivo intrínseco. Escisión que, de ser consecuente, conlleva diferentes modos y contenidos de dicha Matemática: contar, matemática «elementalm, aplicada, pura... Y es el cuadro propio que se ha venido teniendo hasta tiempos muy recientes.

En cualquier caso, y junto a otras materias, la Matemática se ha estimado, según los casos, como el instrumento clave para conducir bien la razón; o para formar eficientes servidores del Estado... Así, antes y durante la Ilustración, la enseñanza de la Matemática podía considerarse útil para "conducir bien la razón" y para que algún filósofo natural dictara clases a las princesas de turno, o al menos dirigiera sus cartas correspondientes, porque esas princesas debfan saber hasta de las nociones de espacio y tiempo - por supuesto newtonianaspara conducir bien los asuntos de Estado. Labor, la de Euler por ejemplo, que rememora en cierto sentido al filósofo de la República platónica. Papeles asignados a la Matemática según los contextos...

En todos los casos, la Matemática como elemento instrumental del conocer cientifico, lenguaje obligado de las ciencias y las técnicas y hasta, en momentos actuales, de las Ciencias Sociales y Humanas... 
* Esta última respuesta es, ciertamente, parcial. Y ello porque normalmente las exigencias estrictamente pragmáticas vienen adornadas por unas fraseologías que ocultan unas ideologías. Fraseologías por las cuáles a la enseñanza de la Matemática se le va a asignar otros papeles u objetivos ahora en el plano pretendidamente educativo y no sólo en el que se considera instrumental social como en las funciones anteriores. Son otros planos que dependen de la visión que se tiene de la Matemática, visión tópica pero que supone una valoración especial.

* Voy a centrarme, como ejemplo, en las funciones u objetivos que se le asignan en momentos como los actuales, en una Ley como la de Educación. En ella, y como objetivos educativos que se asignan a la enseñanza de la Matemática en la ESO, se encuentran los tres siguientes:

1. Valor instrumental en el sentido de que:

las matemáticas proporcionan formalización al conocimiento humano riguroso y, en particular, al conocimiento científico.

2. Aplicación funcional, en el sentido de aplicar los conocimientos matemáticos:

fuera del ámbito escolar, en situaciones de la vida cotidiana

3. Establecimiento de destrezas cognitivas:

susceptibles de ser utilizadas en una amplia gama de casos particulares, y que contribuyen, por si mismas, a la potenciación de las capacidades cognitivas de los alumnos.

Todos los objetivos son, en el fondo, instrumentales. En ningún caso se dice que se enseñe matemática porque sí, por su belleza intrínseca. En todos los casos se la adopta como algo ajeno pero pragmático: utensilio para la vida - para la formalización del conocimiento uriguroso" o para la formación del individuo y esto último supone, también, utensilio para la vida...

En esta concepción se subtienden, implícitas a veces y otras no tan implicitas, unas creencias que considero tópicas en torno a qué sea el Hacer matemático. Voy a mencionar sólo alguna de ellas.

a. Por una parte parece admitirse que la Matemática es el reino del razonamiento deductivo y formalizador por excelencia. El Hacer matemático se muestra como un hacer demostrativo y riguroso. Quien domine este hacer dominará, 
consecuentemente el modo de razonar del geómetra, del matemático y, por ello, dominará el razonamiento deductivo, riguroso. De modo explícito esta idea se enuncia en el Anexo I del BOE 21-10-92, donde se afirma sobre el carácter de las matemáticas:

les caracteriza la naturaleza lógica deductiva de su versión acabada, el tipo de razonamiento que utilizan y la fuerte cohesión interna dentro de cada campo y entre unos campos y otros.

Bien entendido, se refiere a las matemáticas, no a los matemáticos que, según yo pensaba hasta ahora, son los que razonan, pero la Ley es la Ley y según la misma son las matemáticas las que utilizan un tipo de razonamiento determinado.

Desde este enfoque es consecuente que el individuo no tenga que poseer conocimiento matemático alguno sino que se le asigna el construir o elaborar las Matemáticas. Y así, se puede leer:

Participar en el conocimiento matemático consiste, más que en la posesión de los resultados finales de esta ciencia, en el dominio de su (forma de hacer)...

No se trata de que los alumnos posean muchas y sofisticadas herramientas, sino las estrictamente necesarias y que las manejen con destreza y oportunamente.

Dos párrafos que sutilmente se precisan entre sí: dominar las formas de hacer matemática, pero no todas, sólo unas pocas. Tenemos, así, la imagen de una única Matemática de la que los alumnos han de participar en la elaboración, en su construcción o más bien descubrimiento. Participación en una disciplina caracterizada por la naturaleza lógica deductiva de su versión acabada, fuertemente cohesionada en cada campo - sin especificar qué es ese campo-, y también muy cohesionada con otros campos. Y la participación no ha de ser en los conocimientos sino en la forma de hacer.

Consecuentemente, esa forma de hacer no puede ser otra que la naturaleza lógica deductiva. Y quien domine este tipo de naturaleza deductiva lógica dominará la disciplina; aunque no se pretende tanto, sino que se acota el terreno y se indica que debe manejar sólo unas cuantas herramientas, "las estrictamente necesarias" con destreza y oportunidad. Pero en el manejo de esta metáfora "industrial» no se especifica bien a qué denominar "estrictamente necesarias"... 
En cualquier caso, lo que pretendo indicar es, por un lado, la convicción de que a la Matemática se le asigna como carácter básico el de ser una disciplina lógico-deductiva, rigurosa; por otro, el giro inmediato a convertirla en herramienta del razonar, en su visión utilitaria, pragmática.

Y la primera pregunta que hago, en este terreno, es si se puede razonar en abstracto y si el razonar no obliga, siempre, a razonar sobre algo, con un contenido. Razonar sin contenido me parece que lleva a aceptar la existencia de unos moldes llamados "razonamiento formal" que podrán aplicarse, indiscriminadamente, sobre cualquier masa de plastilina. Y si es esto lo que se admite, y se insiste en la metáfora industrial, en el término de herramienta, de instrumento, se olvida que al tomarlo como instrumento razonador, el instrumento elegido depende de la materia sobre la que se va a razonar: no es lo mismo la herramienta de un electricista que la de un losador o la utilizada en una planta de montaje de automóviles... No sé si hay herramientas en abstracto, puramente formales y universales $y$, por ello, aplicables a cualquier tipo de materia...

En segundo lugar, el término "rigurosa» es término algo difuso como para atribuirlo, sin más, a la Matemática. Y ya saben las discusiones constantes en torno a este término.

En tercer lugar, cuando se pasa a aplicar normas como las anteriores, se utilizan términos como los de procesos deductivos de carácter muy elemental como si los procesos deductivos poseyeran diferentes grados que vendrían acompañados, se supone, de diferentes grados de rigor... $\mathrm{O}$, en esa exclusividad de la naturaleza lógico-deductiva de las matemáticas, parece olvidarse que también existen razonamientos por analogía, por ejemplo, que constituyen una de las formas en que se manifiesta la metáfora tan poco admitida en el terreno tópico matemático, aunque se maneje permanentemente para referirse a las matemáticas. Lo cual es claro si de lo que se habla es de "las matemáticas" y no de los matemáticos.

b. El razonar se quiere, además, deductivo y formal. Supone aceptar, como concepción subyacente, la existencia de un único modo de hacer matemática, único modo plasmado desde Euclides y ya para siempre. Una concepción como la que Kant tuviera de la Lógica, que estimaba salida de la cabeza de Aristóteles completa y acabada, sin avances ni retoque alguno, atemporal y válida para todo tiempo y lugar.

Una idea que, desde mi punto de vista, es radicalmente errónea. No voy a mantener, aquí, que no es lo mismo la Matemática que se enseña en la escuela 
- saber contar...- que la que se pueda enseñar en la Politécnica o en la Facultad de ciencias. Voy a algo más profundo. A la afirmación de que existen diferentes tipos de Hacer y que cada uno implica unos modos distintos tanto en su metodología como en su concepción epistemológica.

Hay un Hacer constructivo como el establecido en los Elementos euclídeos que parten del objeto dado y, sobre ese objeto se realizan construcciones, las permitidas por el marco de referencia -y puede verse esta construcción modélica en Kant-y que no suponen carácter derivativo lógico alguno aunque se admita que son «demostraciones»; o las resoluciones de ecuaciones diferenciales a lo d'Alembert donde tampoco aparece el elemento derivativo por ningún lado. Y son demostraciones, y muy rigurosas, pero en su campo propio de Hacer, el Figural, donde el Hacer Matemático aparece, realmente, como un Arte en el sentido primigenio de este término. Como Arte=Técnica, en este Hacer se siguen unos principios o reglas, normas de construción y no principios o postulados de carácter proposicional veritativo-funcional.

A lo largo del s. XIX, y básicamente al final del siglo, se instituye otro tipo de Hacer matemático, el que he denominado Global, donde la magnitud se adopta como distributiva extensional, es decir, como agregado de elementos, como conjunto de elementos cualesquiera. Ello obliga a crear nuevos modos de definición y de demostración, básicamente existenciales porque se parte del agregado o conjunto y no del objeto. Obliga a establecer una nueva ideografía y a plantear cuestiones acerca del rigor y, por supuesto, a modificar la noción de demostración, convertida ahora en derivación a partir de unos primeros principios o postulados veritativo-funcionales, los caracterizadores del campo de juego. Y es el paso a un Hacer matemático de Teorías y estructuras donde la intuición, el objeto dado, desaparecen en un primer momento porque lo que se manejan son conceptos de segundo nivel. Aquí sí rige el papel hipotético-deductivo. Pero bien entendido que tampoco se hacen "derivaciones" lógico-deductivas, sino "demostraciones» que poseen un valor epistémico y no sólo sintáctico básico.

$\mathrm{Y}$ junto a estos tipos de Hacer se ha tenido, desde siempre, pero ahora potenciado, el algorítmico o computacional, con su apoyatura en el cálculo y en la programación, ahora en el ordenador. Un Hacer computacional donde un teorema se ha convertido en un programa y que ha llevado a ciertas exageraciones como a la afirmación de John Horgan en «La muerte de la demostración" 
los ordenadores están transformando las vías para el descubrimiento, la demostración y la comunicación de ideas matemáticas.

Son diferentes haceres que, desde mi punto de vista, se enfrentan en cierta manera a unas versiones tópicas pero que se plasman en la enseñanza de la Matemática. Versiones, en cualquier caso, siempre teñidas de un carácter pragmático por el cual la enseñanza de las Matemáticas se encuentra, siempre, al servicio de...

Una enseñanza de la Matemática que ha entrañado, de modo consecuente, la aparición de los profesionales de la misma y, también consecuente, de los profesionales de las Escuelas Normales - las Escuelas de Magisterio- que enseñen a los primeros. $\mathrm{Y}$, en esa enseñanza, la convicción de que el posible aprendizaje requiere no sólo el dominio de la materia a enseñar sino de enganos, de Didácticas y Metodologías que hagan que el alumno consiga participar en algo ajeno, dado de antemano y como producto acabado y eviten el lema establecido en unos primeros momentos: "la letra, con sangre entra».

Pero ello es claro: todo lo que suponga trabajo y esfuerzo parece suponer, por reacción, huida del mismo. Para evitar la huida hay que enganar al alumno utilizando las Didácticas y Metodologías correspondientes. Lo que ocurre es que estos enganios se atienen, en general, a la convicción señalada acerca de la naturaleza lógico-deductiva de la Matemática, a la existencia, en cada momento, de un único tipo de hacer matemático con unas valoraciones asociadas.

Valoraciones que, en el fondo, condicionan las funciones asignadas a la enseñanza de las matemáticas y a su papel en la sociedad. Son valoraciones que van variando según el tipo de sociedad existente en cada momento. Así, Puig Adam-Rey Pastor en 1933, mantenían el aspecto de formación humanística de la enseñanza de la Matemática junto al amor a la naturaleza, el sentido de la observación... Ahora se mantiene en función de los tres objetivos o funciones puramente instrumentales antes señalados.

Curiosamente Didáctica y Metodología sólo para los primeros niveles de esa enseñanza. Parece admitirse que en las Grandes Escuelas, en la Universidad, o no hay que engañar a nadie o la deformación es ya tan enorme que se admite lo que le den sin protesta alguna.

En este punto se parte de otro tópico avalado por corrientes subyacentes de Empirismo y Psicologismo: el establecido en las llamadas a la abstracción —de la que nunca se sabe muy bien en qué consiste-. Supone, en cualquier caso, 
partir de lo concreto para llegar al concepto abstracto como si este mecanismo fuera lo psicológico «natural». Se admite, sin más, que los conceptos clasificatorios se obtienen abstrayendo a partir de objetos concretos: "mesa" a partir de observar muchas mesas. No se tiene en cuenta, entre otras cosas, el uso contextual del lenguaje que hace que dispongamos del término "mesa" y se aplique cada vez que se oberve una mesa. Uso contextual que lleva a considerar que es la previa clasificación la que permite "percibir» los objetos de una u otra manera.

El carácter proteico del Hacer matemático ha quedado en la enseñanza un tanto difuminado en beneficio de sólo alguno de sus aspectos, el instrumental. Enseñanza de pretendidas formas de razonamiento y métodos sin contenido que llevan a que el ciudadano actual sea un individuo «anumérico" que no sepa calcular aunque esté inundado de cifras pero sí pueda explicar "qué es calcular". Incluso se ha llegado a que alumnos universitarios, estudiantes de Matemáticas en Cuarto Curso, puedan afirmar que no saben el valor del área del círculo y que tendrían que obtenerlo integrando... 